\title{
EUROfusion
}

EUROFUSION WPMST1-PR(16) 15803

E Viezzer et al.

\section{Investigation of inter-ELM ion heat transport in the H-mode pedestal of ASDEX Upgrade plasmas}

Preprint of Paper to be submitted for publication in Nuclear Fusion

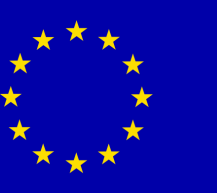

This work has been carried out within the framework of the EUROfusion Consortium and has received funding from the Euratom research and training programme 2014- 
This document is intended for publication in the open literature. It is made available on the clear understanding that it may not be further circulated and extracts or references may not be published prior to publication of the original when applicable, or without the consent of the Publications Officer, EUROfusion Programme Management Unit, Culham Science Centre, Abingdon, Oxon, OX14 3DB, UK or e-mail Publications.Officer@euro-fusion.org

Enquiries about Copyright and reproduction should be addressed to the Publications Officer, EUROfusion Programme Management Unit, Culham Science Centre, Abingdon, Oxon, OX14 3DB, UK or e-mail Publications.Officer@euro-fusion.org

The contents of this preprint and all other EUROfusion Preprints, Reports and Conference Papers are available to view online free at http://www.euro-fusionscipub.org. This site has full search facilities and e-mail alert options. In the JET specific papers the diagrams contained within the PDFs on this site are hyperlinked. 


\title{
Investigation of inter-ELM ion heat transport in the H-mode pedestal of ASDEX Upgrade plasmas
}

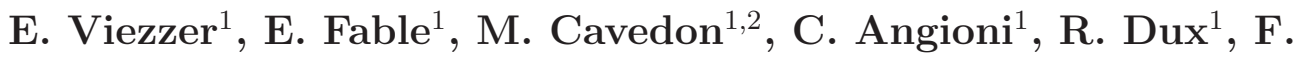 \\ M. Laggner ${ }^{3}$, M. Bernert ${ }^{1}$, A. Burckhart ${ }^{1}$, R. M. McDermott ${ }^{1}$, \\ T. Pütterich ${ }^{1}$, F. Ryter ${ }^{1}$, M. Willensdorfer ${ }^{1}$, E. Wolfrum ${ }^{1}$, the \\ ASDEX Upgrade Team and the EUROfusion MST1 Team $\ddagger$ \\ 1 Max Planck Institute for Plasma Physics, Boltzmannstr. 2, 85748 Garching, \\ Germany \\ 2 Physik-Department E28, Technische Universität München, 85748 Garching, \\ Germany \\ 3 Institute of Applied Physics, TUWien, Fusion@ÖAW, Wiedner Hauptstr. 8-10, \\ 1040 Vienna, Austria \\ E-mail: Eleonora.Viezzer@ipp.mpg.de
}

\begin{abstract}
The ion heat transport in the pedestal of H-mode plasmas is investigated in various $\mathrm{H}$-mode discharges with different pedestal ion collisionalities. Interpretive modelling suggests that in all analyzed discharges the ion heat diffusivity coefficient, $\chi_{i}$, in the pedestal is close to the neoclassical prediction within the experimental uncertainties. The impact of changing the deposition location of the electron cyclotron resonance heating on the ion heat transport has been studied. The effect on the background profiles is small. The pre-ELM (Edge Localized Modes) edge profiles as well as the behaviour of the electron temperature and density, ion temperature and impurity toroidal rotation during the ELM cycle are very similar in discharges with onand off-axis ECRH heating. No significant deviation of $\chi_{i}$ from neoclassics is observed when changing the ECRH deposition location to the plasma edge.
\end{abstract}

PACS numbers: 52.55.Fa, 52.25.Vy, 52.30.-q, 52.70.-m

\section{Introduction}

The gradients in the ion and electron temperature profiles are a key component for driving turbulent transport in plasmas. Since the 1980s, 'profile resilience' has been observed on many tokamaks. 'Stiff' temperature profiles describe the fact that the ion and electron temperature profiles, $T_{i}$ and $T_{e}$, are limited by a critical normalized temperature gradient $R / L_{T}=R|\nabla T| / T$, with $R$ the major radius and $\nabla T$ the radial temperature gradient. Beyond this critical $R / L_{T}$ the heat diffusivities increase drastically $[1,2]$. Hence, with stiff profiles the edge temperature is a key to attaining higher core temperatures and, therefore, higher confinement of the plasma. The physical $\ddagger$ See http://www.euro-fusionscipub.org/eu-im 
mechanism of the core profile resilience is plasma turbulence driven by gradients, such as ion temperature gradient modes, trapped electron modes and electron temperature gradient modes. Another contribution to the outward heat flux is induced by Coulomb collisions described by neoclassical theory [3], which includes the effects of the magnetic geometry. Both contributions can be described as purely diffusive assuming locality. Transport due to turbulence leads to electron and ion heat diffusivities that strongly exceed the neoclassical prediction, which is typically observed in tokamak plasmas. In the pedestal region of $\mathrm{H}$-mode plasmas, however, turbulent particle transport is strongly reduced due to the existence of steep radial gradients in the electric field perpendicular to the magnetic field [4]. Understanding the transport processes in the H-mode transport barrier is essential for a reliable scaling to next step fusion devices.

Extensive studies of the heat transport in the plasma core have been carried out [5, $6,7,8,9,10]$, however, little focus was directed to the pedestal region due to lack of high-resolution diagnostics. In the past years extensive effort has been directed to upgrading the diagnostics at the plasma edge in order to resolve the fast dynamics and the steep gradients that are present in the pedestal region [11]. Previous analysis of the edge temperature gradients measured at ASDEX Upgrade (AUG) showed that the pedestal ion heat transport coefficient in-between ELMs is consistent with neoclassical theory $[12,13]$. A dedicated discharge was selected for the modelling and the measured temperature profile was compared to simulations using the 2D fluid code B2.5 which showed good agreement within the experimental uncertainties. A detailed pedestal plasma transport benchmarking comparison between various codes was performed at DIII-D [14]. It was found that the effective ion heat diffusivity profile differs from the neoclassical prediction and may be even smaller than it. Theoretical studies dedicated to the kinetic effects on the ion heat transport [15] and the impact of finite banana effects on the neoclassical ion thermal conductivity [16] showed that such effects could be quite important in the pedestal. However, comparison to experimental data are still pending.

In this work, the edge ion heat transport is studied using power balance analysis with the transport code ASTRA. For the analysis dedicated discharges at low collisionality, where the ion and electron heat channels are not too strongly coupled, were selected, which are introduced in section 2. The analysis methods for modelling the ion heat transport are presented in section 3. A comparison between the experimentally determined heat transport coefficient and neoclassical predictions shows good agreement in the edge transport barrier region (see section 4). A summary of this paper is given in section 5 .

\section{Experiments}

For the evaluation of the heat transport coefficients a good separation between electron and ion heat flux is required, i.e. at low collisionality where the electron-ion energy exchange term in the power balance is negligible or when the difference between $T_{e}$ and $T_{i}$ is larger than the uncertainties. 


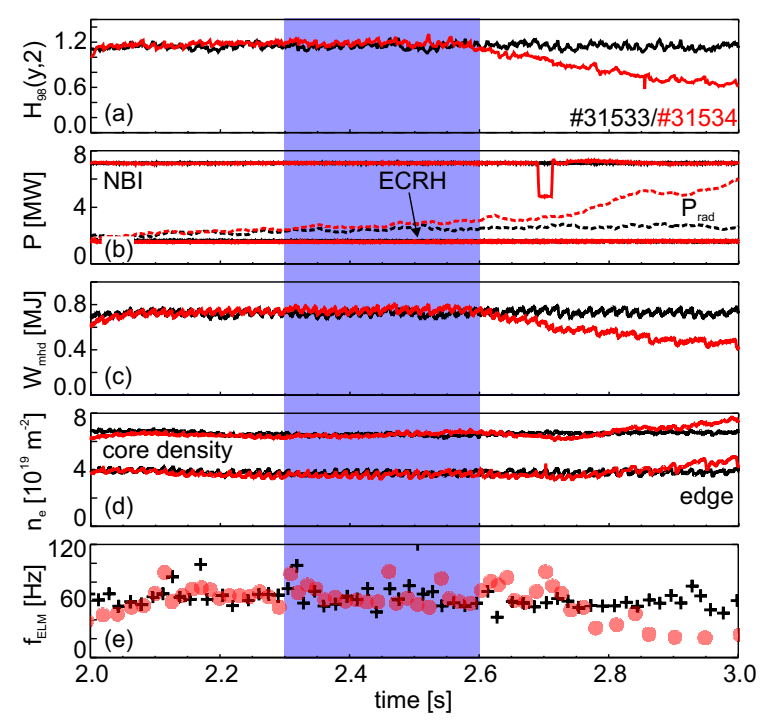

Figure 1: Time traces of H-mode discharges \#31533 (black) and \#31534 (red): (a) global energy confinement factor $H_{98}(y, 2)$, (b) NBI power, ECRH power and radiated power $P_{r a d},(\mathrm{c})$ stored energy $W_{m h d}$, (d) line-integrated electron density $n_{e}$ in the plasma core and edge and (e) ELM frequency $f_{E L M}$.

Dedicated discharges at low collisionality were carried out in the ASDEX Upgrade (AUG) tokamak. In these H-mode plasmas the plasma current was $1 \mathrm{MA}$, the toroidal magnetic field $B_{t}=-2.47 \mathrm{~T}$, the edge safety factor $q_{95}=4.3$, the core line-integrated density varied from $6.3-7.5 \times 10^{19} \mathrm{~m}^{-2}$, and the total heating power was $8.7 \mathrm{MW}$, i.e. 7.1 MW neutral beam injection (NBI) and 1.6 MW electron cyclotron resonance heating (ECRH). Low density plasmas usually facilitate to have a large difference between ion and electron temperature profiles. Despite rather low D gas puff rates of $1.6-$ $3.1 \times 10^{21} \mathrm{~s}^{-1}$ the edge line-integrated electron density is roughly $4 \times 10^{19} \mathrm{~m}^{-2}$ due to the fuelling of the plasma by NBI. The collisionality in these plasmas varied from $0.2-0.5$ in the pedestal region $\left(\rho_{p o l}=0.97\right)$. Note that here the ion collisionality is defined as the effective collision frequency divided by the trapped particle bounce frequency, $\nu_{i}^{*}=$ $\nu_{\text {eff }} / \omega_{\mathrm{b}}=\nu q R /\left(\epsilon^{3 / 2} v_{\mathrm{th}}\right)$. The triangularity of the plasma was $\delta \sim 0.31$, while the ELM frequency was constant at around $60 \mathrm{~Hz}$ (see figure 1(d)). The ECRH scheme was also changed from core to edge deposition to study the possible impact on the edge plasma profiles and on the ion heat transport which is presented below.

The time traces of two example discharges are illustrated in figure 1. Figure 1 (a) shows the energy confinement factor $H_{98}(y, 2)$, (b) the NBI power, ECRH power and radiated power $\left(P_{r a d}\right),(\mathrm{c})$ stored energy $W_{m h d},(\mathrm{~d})$ core and edge line-integrated density and (e) the ELM frequency $f_{E L M}$. The black time traces correspond to the on-axis ECRH case (\#31533), while the red lines to the discharge with off-axis ECRH deposition (\#31534). The high-resolution diagnostic suite available at AUG enables the measurement of the plasma profiles, such as ion temperature $\left(T_{i}\right)$ and impurity toroidal rotation velocity $\left(v_{t o r}\right)$ as well as electron temperature $\left(T_{e}\right)$ and density $\left(n_{e}\right)$ with high temporal and radial 
resolution. The $T_{i}$ and $v_{\text {tor }}$ profiles are measured using charge exchange recombination spectroscopy on a heating beam [17]. The electron density profile is measured with laser interferometry [18], impact excitation on a Lithium beam [19] and Thomson scattering [20]. The Thomson scattering diagnostics also provides information on $T_{e}$ which is complemented by measurements with the electron cyclotron emission (ECE) diagnostic [21]. Note that $T_{e}$ and $n_{e}$ can be evaluated with the integrated data analysis (IDA) package using Bayesian probability theory [22], which combines data from different diagnostics, i.e. Lithium beam spectroscopy, ECE and interferometry. The IDA evaluation is based on a forward model which includes information from various diagnostics taking into account the corresponding uncertainties. The combined analysis enables the simultaneous analysis of $n_{e}$ and $T_{e}$ with a temporal resolution down to $250 \mu \mathrm{s}$. Example profiles of the ion and electron temperature, electron density and toroidal rotation are presented in the following subsection.

The transport analysis was extended to plasmas with varying collisionality. Note, however, that at higher collisionality the determination of the ion heat flux and thus, $\chi_{i}$ has a significantly larger error bar as the electron ion heat exchange is not negligible due to a stronger coupling between ions and electrons. All discharges feature a low ELM frequency $(\leq 100 \mathrm{~Hz})$ to allow for an inter-ELM evaluation of the ion heat diffusivity. The parameter range of the discharges included in the analysis are: $I_{p}=0.8-1.05 \mathrm{MA}, B_{t}$ $=2.46-2.51 \mathrm{~T}, q_{95}=3.7-5.25$, core line-integrated density of $5.5-7.6 \times 10^{19} \mathrm{~m}^{-2}$, pedestal ion collisionalities (at $\rho_{\text {pol }}=0.97$ ) of $0.21-2.43$ and normalized beta $\beta_{N}$ of $0.95-2.12$. All discharges were carried out in deuterium plasmas with ECRH and NBI heating.

\subsection{On- and off-axis ECRH heating}

One method to get a better understanding of the transport processes is to determine how the transport is affected if the deposition location of the heat input is displaced from the magnetic axis of the plasma [7]. Changing the deposition location from the core to the edge of the plasma can affect the plasma $\beta$ and thus, the stability of the pedestal. As shown in figure 2 the magnetic axis and the flux surfaces are slightly displaced when applying edge ECRH heating. This also allows to test whether the assumption of locality is applicable or whether the heat transport in the pedestal can be influenced by the core. The goal of the experiments presented in this subsection is to test whether an edge deposition of the ECRH power has an impact on the behaviour of the plasma profiles during the ELM cycle and on the ion heat transport. Previous experiments on AUG showed that the application of edge ECRH heating affected the ELM frequency and caused a small relaxation of the edge gradients, while the MHD stability boundary remained unchanged [23]. A second, higher frequency ELM band appeared when depositing more ECRH power to the edge. This is in contrast to results from TCV [24] which showed an increase of the ELM frequency by a factor of two with edge ECRH heating, while the energy loss per ELM was reduced.

Figure 2 shows the location of the ECRH power deposition and compares the (a) on- and 

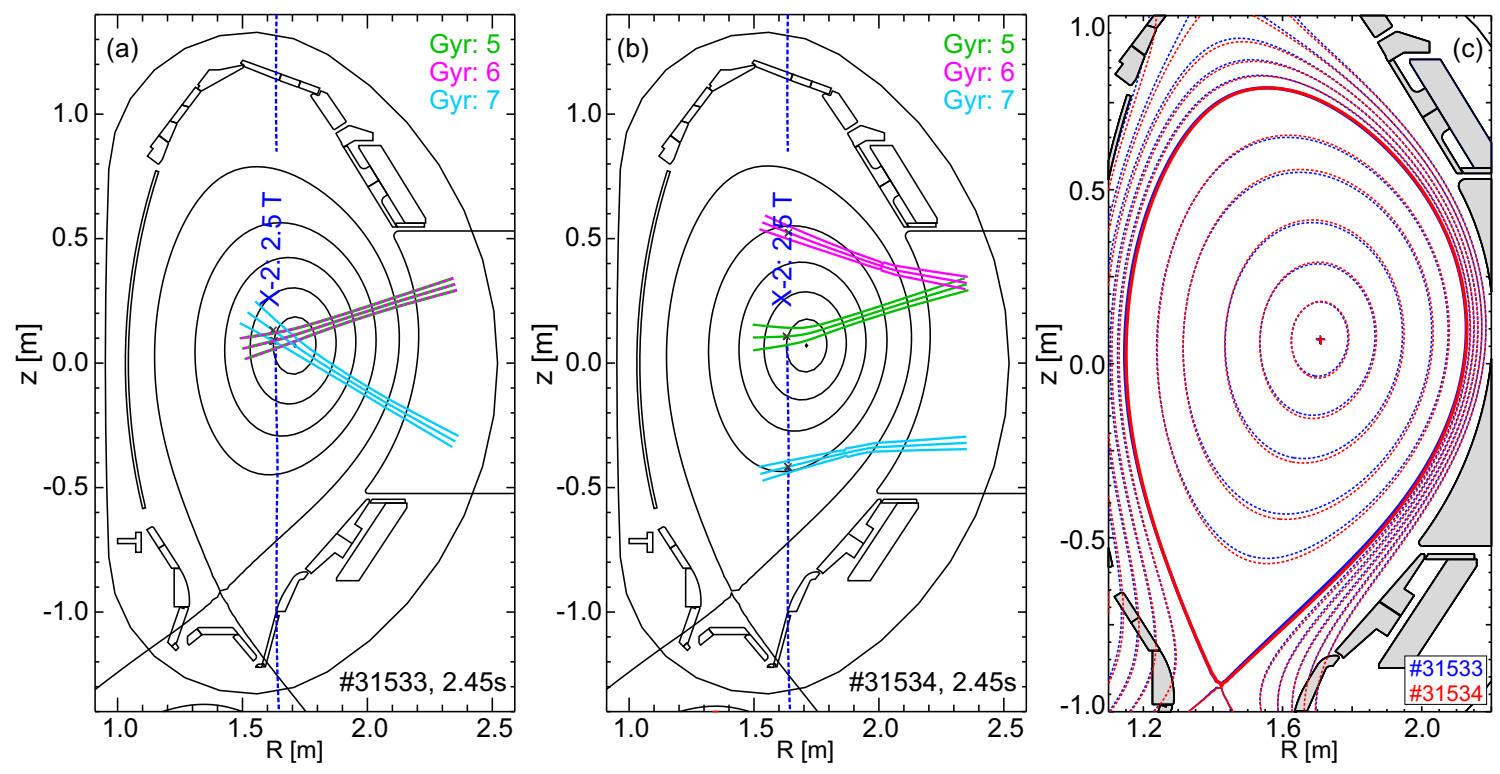

Figure 2: ECRH deposition location for (a) \#31533 and (b) \#31534; (c) comparison of magnetic equilibria of both cases.

(b) off-axis cases calculated using the beam tracing code TORBEAM [25]. Figure 2(c) shows the magnetic equilibria of both discharges (on-axis case in blue and off-axis case in red). A total of 1.6 MW heating was provided by three gyrotrons. For the off-axis case, one gyrotron was kept on-axis to avoid impurity accumulation in the plasma core and all the other parameters such as gas puff rate were kept the same. The density of the ECRH power deposition is shown in figure 3. Note the difference in the scale when comparing the absolute numbers. In figure 3 the power density is plotted in $\mathrm{MW} / \mathrm{m}^{3}$ and therefore, the edge heating scenario appears to deposit less power than the on-axis case since the volume unit is larger at the edge. Note, however, that the amount of power input remained unchanged. As shown in figure 1, at these medium density levels the edge ECRH has hardly an impact on the plasma parameters, including the ELM frequency. Note that in [23] the deposition location could be moved even further towards the plasma edge, i.e. $\rho_{\text {pol }} \approx 0.88$.
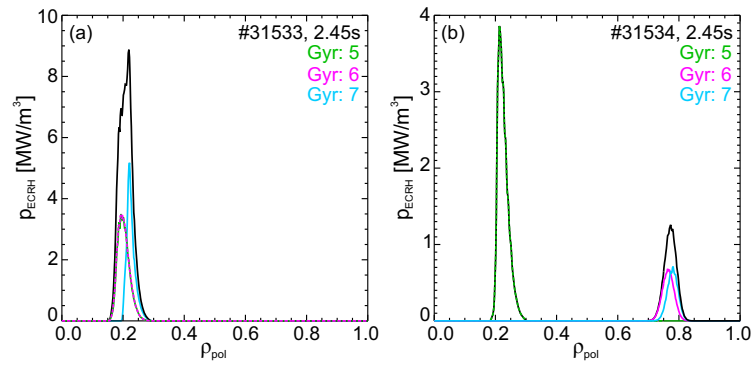

Figure 3: ECRH power deposition density of (a) on-axis case and (b) off-axis case. Note that the scales of the y-axis are different. 

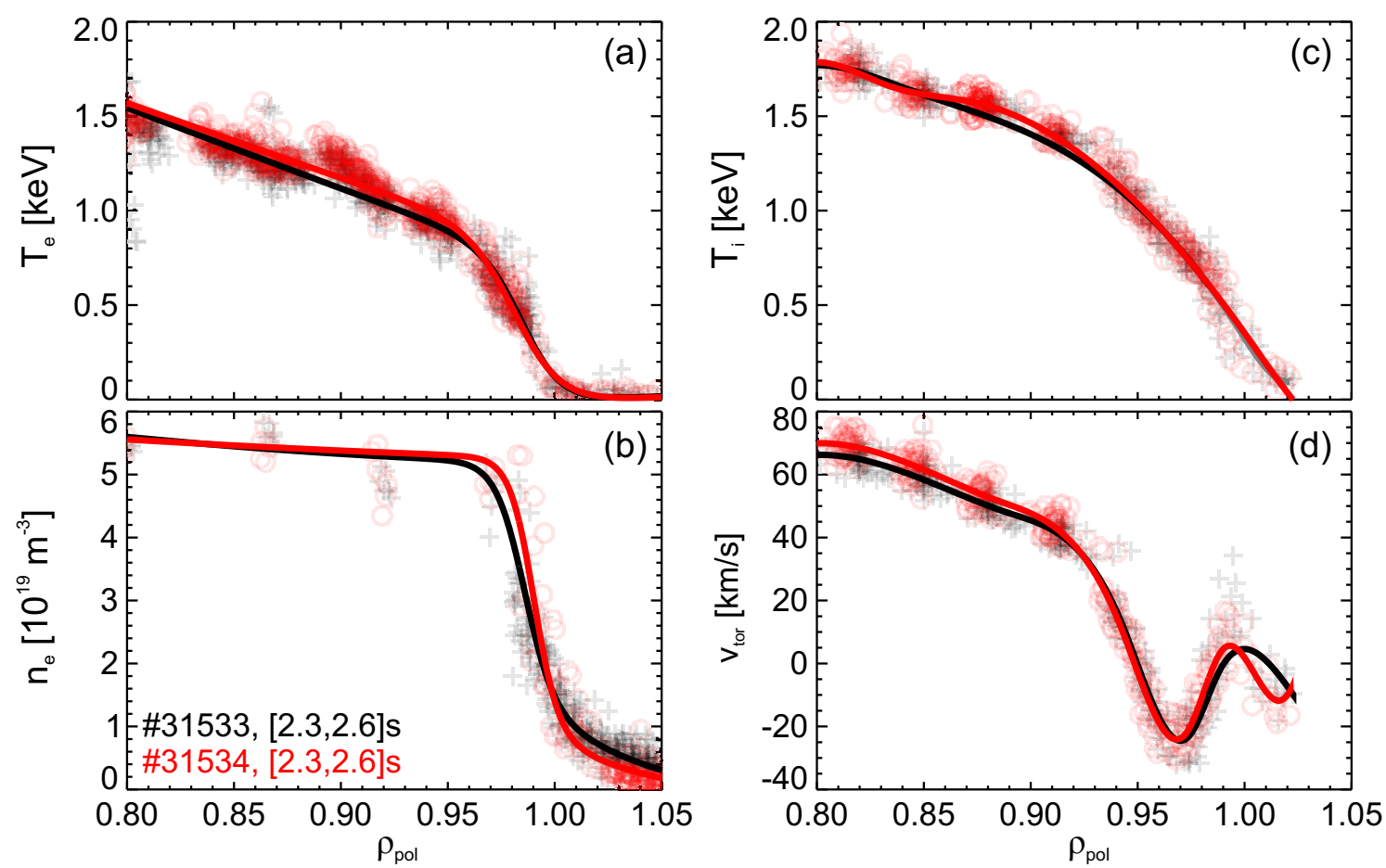

Figure 4: Pre-ELM profiles of (a) $T_{e}$, (b) $n_{e}$, (c) $T_{i}$ and (d) $v_{\text {tor }}$. Discharge \#31533 (on-axis ECRH) is shown in black, while \#31534 (off-axis ECRH) is shown in red. The solid lines are fits to the measured data points (modified tanh function for $T_{e}$ and $n_{e}$ and spline fits for $T_{i}$ and $\left.v_{t o r}\right)$.

Figure 4 shows the profiles of the (a) electron temperature and (b) density, (c) ion temperature and (d) toroidal rotation for the on-axis (black) and off-axis (red) ECRH case. The measured data points are marked by circles and crosses, while the solid lines correspond to the fits (spline fits for $T_{i}$ and $v_{t o r}$, modified hyperbolic tangent function for $T_{e}$ and $n_{e}$ ). The edge profiles are hardly changed by applying the deposition location to the plasma edge $\left(\rho_{\text {pol }} \approx 0.78\right)$ and the gradients are very similar within the experimental uncertainties.

The behaviour of the profile recovery during the ELM cycle is shown in figures 5, 6 and 7. In figures 5 and 6 the evolution of the profiles is shown at three radial positions in the pedestal region, $\rho_{\text {pol }}=0.95$ (black and red), 0.97 (blue and magenta) and 0.99 (turquoise and orange). The measured data points are marked by diamonds and circles, while the lines represent the mean average during a time interval of $1 \mathrm{~ms}$ (for the electron temperature and density) and $2 \mathrm{~ms}$ (for the ion temperature), respectively. The solid lines correspond to the on-axis ECRH case, while the dashed lines represent the discharge with off-axis ECRH deposition. For the evolution of $T_{e}$ and $n_{e}$ presented in figures 5 and 6 the measurements are evaluated using the IDA technique with a temporal resolution of $250 \mu \mathrm{s}$. Note that for these discharges the temporal resolution of the edge CXRS diagnostics is limited to $2.3 \mathrm{~ms}$. While the behaviour of the CXRS profiles during the ELM crash cannot be resolved, the behaviour during the recovery phases can be 
Investigation of inter-ELM ion heat transport in the H-mode pedestal of ASDEX Upgrade7

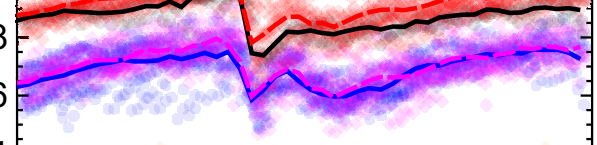



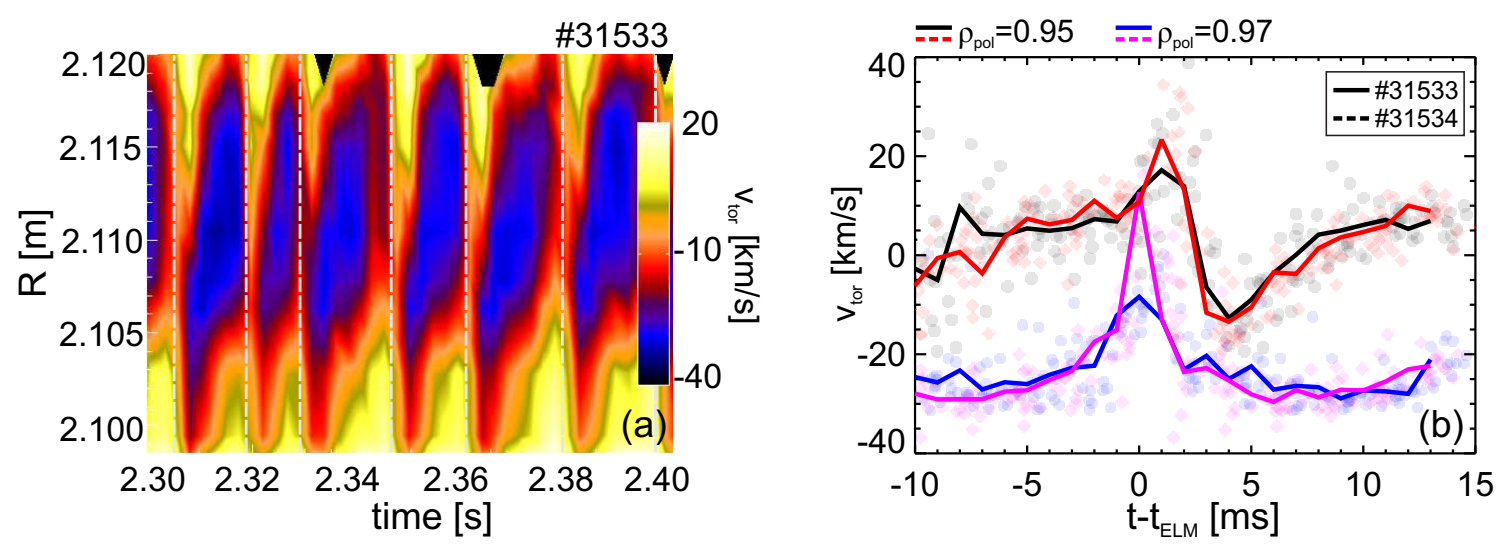

Figure 7: Behaviour of the toroidal rotation profile during the ELM cycle: (a) Contour plot of the measured data, (b) temporal evolution of $v_{\text {tor }}$ at $\rho_{\text {pol }}=0.95$ and 0.97 .

\section{Transport analysis methods}

The ion heat conductivities are determined using power balance analysis with ASTRA [28], a 1.5D transport code, which solves the time dependent and flux surface averaged heat transport equation. Within ASTRA the equilibrium is reconstructed with a prescribed boundary condition and a 3-moment approach. Initial profiles for $T_{e}$ and $T_{i}$ are used as input, while a time-dependent boundary condition for $T_{e}$ and $T_{i}$ at the separatrix can be used. Measurements of the $n_{e}$ profile and the radiated power density $\left(P_{\text {rad }}\right)$ are also provided as input. The ion and electron energy sources as well as the heat exchange between ions and electrons are evaluated which determine the electron and ion heat fluxes $Q_{e}$ and $Q_{i}$.

In the simulations the electron heating power consists of the ohmic heating power $\left(P_{o h}\right)$, the collisional heat exchange rate between ions and electrons $\left(P_{e i}\right)$, the power lost as radiation $\left(P_{r a d}\right)$, power losses due to atomic processes $\left(P_{e}^{N}\right)$ and the auxiliary power to the electrons $\left(P_{e, \text { aux }}\right)$. The ion energy source depends on the electron-ion exchange rate, losses due to atomic processes and the auxiliary ion heating such as neutral beam injection:

$$
\begin{aligned}
& P_{e}=P_{\text {oh }}-P_{e i}-P_{\text {rad }}-P_{e}^{N}+P_{e, a u x} \\
& P_{i}=P_{e i}+P_{i}^{N}+P_{i, a u x}
\end{aligned}
$$

The ion and electron energy sources determine the ion and electron heat fluxes related to the temperature gradient via the heat conductivity, $Q=-n \chi(\rho) \frac{\partial T}{\partial \rho}$. In steady state the heat flux corresponds to the integral of the sources, $Q=\int P d V$, where $V$ is the plasma volume. In the following, $\chi_{i}$ is determined experimentally using power balance analysis. To this end, ASTRA is used in an interpretive way. The code can also be run in a predictive way, i.e. using a prescribed $\chi_{i}$ profile and solving the heat transport equations for $T_{e}$ and $T_{i}$. The experimentally determined $\chi_{i}$ is compared to neoclassical predictions in order to test whether neoclassical theory can account for the ion heat transport level in the pedestal. 


\section{Modelling of inter-ELM ion heat transport and comparison to neoclassical theory}

The neoclassical ion heat conductivity is based on the flux surface averaged transport matrix and depends on the density, the poloidal ion gyroradius $\rho_{i, p o l}$ divided by the collisional time $\tau_{i}$, the magnetic field and the neoclassical function $F$ which depends on the trapped particle fraction $f_{t}[29]$ :

$$
\chi_{i}^{\text {neo }}=\frac{n_{i} \rho_{i, p o l}^{2}}{\tau_{i}}\left(\frac{\partial \Psi}{\partial \rho}\right)^{2} \frac{B_{0}^{2}}{\left\langle B^{2}\right\rangle}\left[-F_{22}\left(f_{t}\right)\right]
$$

The experimental ion heat diffusion coefficients are compared to the neoclassical predictions using the NEOART code [30]. For selected discharges they are also compared to simulations with NEO [31] and NCLASS [32]. Figure 8 shows (a) $T_{e}$ (black) and $T_{i}$ (red), (b) the electron (black) and ion (red) energy sources as well as the electronion heat exchange rate (blue) and (c) the resulting surface-integrated heat fluxes as determined via power balance. Note that for the power balance analysis presented in figure 8 the effects due to an ELM have been excluded, i.e. only data taken $-5 \mathrm{~ms}$ to $-1 \mathrm{~ms}$ before the onset of an ELM are used. Figure 9 shows the resulting ion heat diffusion coefficient at the plasma edge. The neoclassical prediction simulated with NEOART is shown in red, while in blue and green the NEO and NCLASS calculations are shown. The power balance analysis yields an edge ion heat conductivity on the order of $\sim 0.6-$ $0.7 \mathrm{~m}^{2} / \mathrm{s}$. Note that the temporal evolution of $T_{i}$ within the analyzed time window is taken into account for the evaluation of the heat flux and the $\chi_{i}^{P B}$ profile shown in figure 9 corresponds to the temporal average, i.e.:

$$
\chi_{i}^{P B}=-\frac{625\left(Q_{i}^{t o t}-Q_{i}^{d t}\right)}{n_{i} \nabla T_{i} \partial V / \partial \rho\left\langle(\nabla \rho)^{2}\right\rangle}
$$

where $Q_{i}^{\text {tot }}$ is the total heat flux, $Q_{i}^{d t}$ the heat flux corresponding to the time variation in the plasma energy, $V$ is the volume, $\rho$ is an effective minor radius defined as $\rho=\sqrt{\Phi / \pi B_{0}}$ with $\Phi$ the toroidal magnetic flux and $B_{0}$ the vacuum magnetic field. \langle\rangle denotes the flux surface average. In the transport barrier region, the ion heat
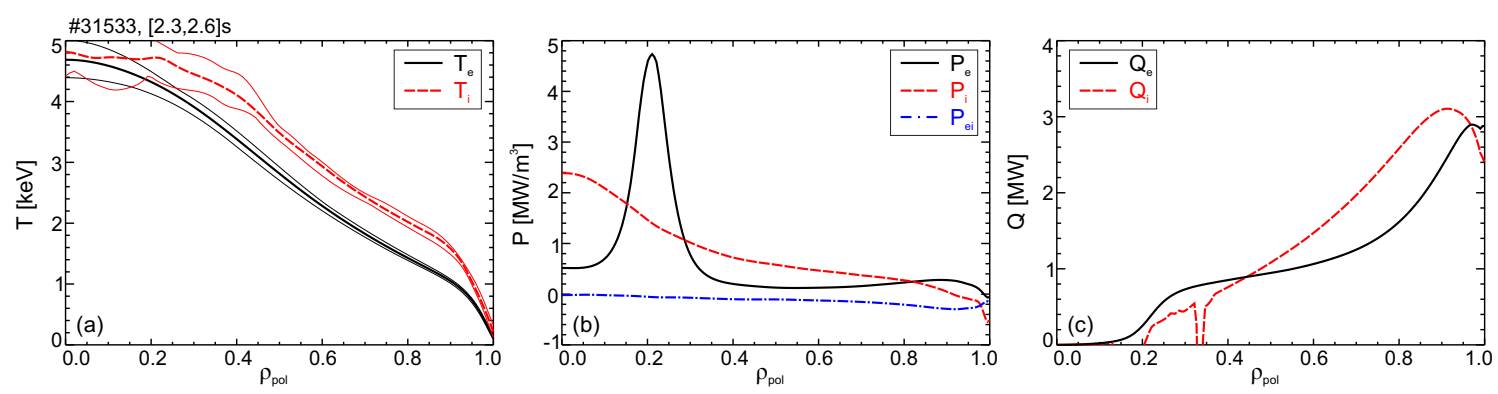

Figure 8: Power balance analysis for discharge \#31533. (a) $T_{e}$ (black) and $T_{i}$ (red) profiles, (b) electron (black) and ion (red) energy sources and electron-ion heat exchange rate, (c) surface-integrated electron (black) and ion (red) heat flux. 


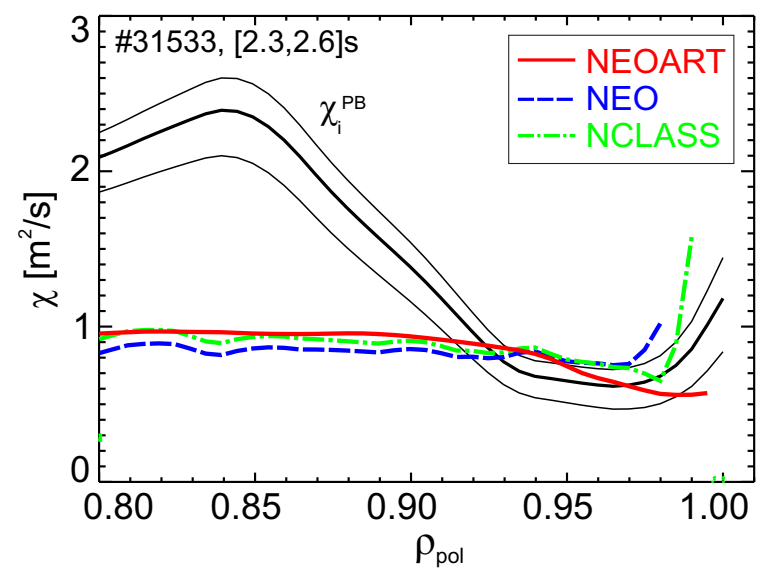

Figure 9: Ion heat diffusivity as determined via power balance $\left(\chi_{i}^{\mathrm{PB}}\right)$ in black, neoclassical predictions calculated with NEOART (red), NEO (blue, dashed line) and NCLASS (green, dashed-dotted line).

conductivity is close to the neoclassical level during the inter-ELM phase, while further inside $\left(\rho_{\text {pol }}<0.9\right) \chi_{i}^{P B}$ exceeds the neoclassical value by a factor of $\sim 2$. Note that close to the separatrix $\chi_{i}^{P B}$ increases again, in qualitative agreement with the predictions by NEO and NCLASS.

The analysis was extended to high collisionality plasmas, i.e. $\nu_{i}^{*}>1$. At higher collisionality, the ion and electron channels are coupled and the electron-ion heat exchange rate is not negligible, making it more difficult to determine the ion heat flux and thus, $\chi_{i}$. Figure 10 shows the ion heat diffusion coefficient for an H-mode plasma with $I_{p}=1 \mathrm{MA}, B_{t}=2.47 \mathrm{~T}$, core line-integrated density of $7.1 \times 10^{19} \mathrm{~m}^{-2}$ and a collisionality of 1.45. Note that here the ELM frequency was $\sim 75 \mathrm{~Hz}$. As shown in figure 10, the edge $\chi_{i}$ remains close to the neoclassical value within the experimental uncertainties also at

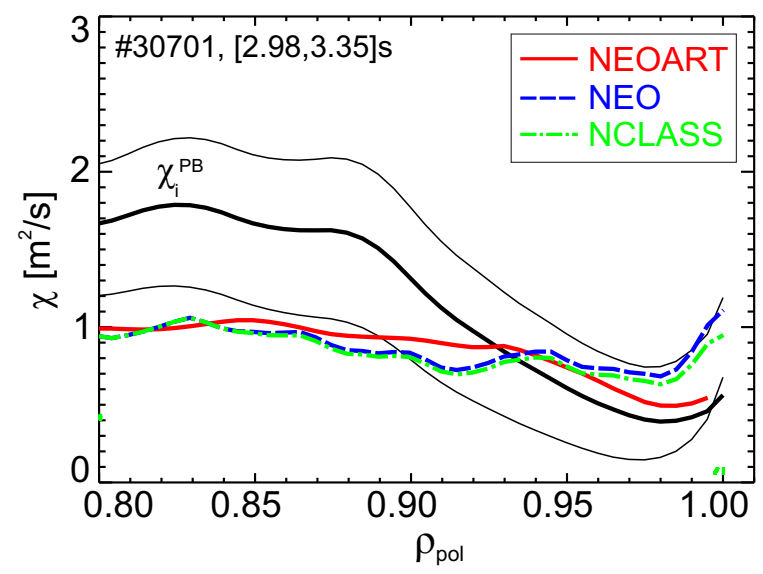

Figure 10: Ion heat diffusivity as determined via power balance $\left(\chi_{i}^{\mathrm{PB}}\right)$ in black, neoclassical predictions calculated with NEOART (red), NEO (blue, dashed line) and NCLASS (green, dashed-dotted line). 

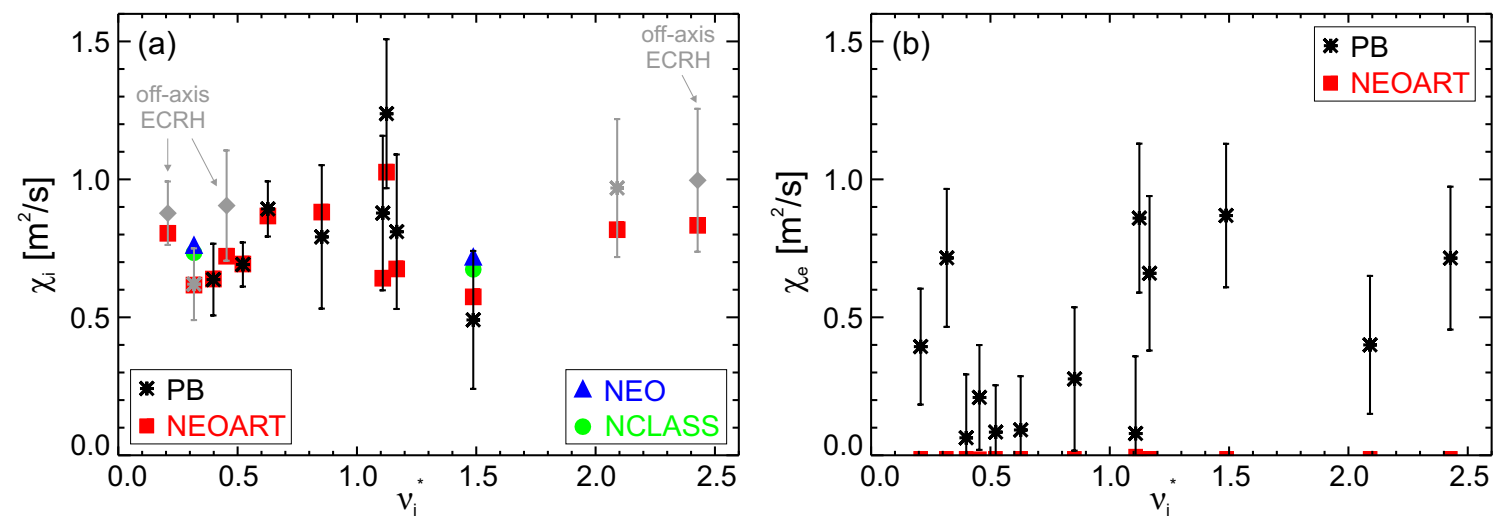

Figure 11: (a) Ion heat diffusivity as a function of the main ion collisionality (at $\rho_{\text {pol }}$ $=0.97): \chi_{i}^{P B}$ determined via power balance analysis in black (stars), neoclassical predictions calculated with NEOART (red, squares), NEO (blue, triangles) and NCLASS (green, circles). Note that the pairs corresponding to the on- and off-axis ECRH cases (stars and diamonds, respectively) are highlighted in gray. (b) Electron heat heat diffusivity plotted versus main ion collisionality (at $\left.\rho_{p o l}=0.97\right): \chi_{e}^{P B}$ determined via power balance analysis in black (stars), neoclassical calculations with NEOART in red (squares).

higher collisionality, in agreement with earlier studies $[12,13]$. Again, further inside $\chi_{i}$ exceeds the neoclassical prediction.

Figure 11(a) shows the pedestal ion heat conductivity as a function of the main ion collisionality. Both values are taken at $\rho_{\text {pol }}=0.97$. The experimentally determined values of $\chi_{i}^{P B}$ are shown in black, while the neoclassical predictions calculated with NEOART are shown in red. For the two cases discussed above, the NEO and NCLASS values are shown in blue and green, respectively. The gray diamonds highlight the $\mathrm{H}$ mode plasmas with edge ECRH deposition, while the corresponding on-axis cases are marked by gray stars. As expected from the background plasma profiles, the ion heat conductivity is hardly changed compared to the on-axis ECRH case. This suggests that despite changing the ECRH deposition location, the edge $\chi_{i}$ remains close to the neoclassical level, at both low and high collisionality. For a comparison, figure 11(b) shows the electron heat transport coefficients as determined via power balance analysis (black stars). Note that for $\chi_{e}$ the evaluation depends on the radiated power, which has large uncertainties. The neoclassical values of $\chi_{e}$ are marked as red squares. While the ion heat transport level is at the neoclassical level, the electron heat transport exceeds the neoclassical predictions by one to two orders of magnitude.

\section{Summary}

Analysis of the inter-ELM ion heat transport in ASDEX Upgrade H-mode plasmas reveals that the ion heat diffusion coefficient is close to the neoclassical level at both low and high collisionality. Dedicated discharges at low collisionality were carried out 
in order to have a small electron-ion heat exchange rate and to have a good separation between $Q_{e}$ and $Q_{i}$. In all analyzed discharges, the experimentally determined $\chi_{i}$ in the edge transport barrier region is in agreement with the neoclassical value during the inter-ELM phase.

The analysis was extended to discharges with varying collisionality and indicates that also at higher collisionality the edge $\chi_{i}$ remains close to the neoclassical value, in agreement with earlier studies $[12,13]$.

The ECRH deposition location was moved from the plasma core to the edge to analyze its impact on the ion heat transport. The off-axis ECRH deposition hardly changes the edge profiles. The behaviour in the recovery of $T_{e}, n_{e}$ and $T_{i}$ during the ELM cycle is also very similar between on- and off-axis heating. The evaluation of the ion heat transport shows no change in the transport level, as expected, indicating that in-between the ELMs the ion heat transport in the edge transport barrier is set by neoclassical diffusion. This also confirms that the assumption of locality can be used for the heat transport in the pedestal region.

\section{Acknowledgments}

This work has been carried out within the framework of the EUROfusion Consortium and has received funding from the Euratom research and training programme 20142018 under grant agreement number 633053. The views and opinions expressed herein do not necessarily reflect those of the European Commission. The support from the EUROfusion Researcher Fellowship programme under grant number WP14-FRFIPP/Viezzer is gratefully acknowledged. F. M. Laggner is a fellow of the Friedrich Schiedel Foundation for Energy Technology.

\section{References}

[1] A. M. Dimits et al. Phys. Plasmas, 7:969, 2000.

[2] A. G. Peeters et al. Phys. Plasmas, 12:022505, 2005.

[3] F. L. Hinton and R. D. Hazeltine. Rev. Mod. Physics, 48(2):239, 1976.

[4] H. Biglari et al. Phys. Fluids B, 2:1, 1990.

[5] P. Gohil et al. Nucl. Fusion, 38(3):425, 1998.

[6] F. Ryter et al. Phys. Rev. Lett., 86(24):5498, 2001.

[7] F. Ryter et al. Nucl. Fusion, 43:1396, 2003.

[8] H. Urano et al. Nucl. Fusion, 48:045008, 2008.

[9] P. Mantica et al. Phys. Rev. Lett., 102:175002, 2005.

[10] F. Ryter et al. Nucl. Fusion, 51:113016, 2011.

[11] E. Wolfrum et al. Nucl. Fusion, 55:053017, 2015.

[12] A. V. Chankin et al. Plasma Phys. Control. Fusion, 48:839, 2006.

[13] E. Wolfrum et al. Conf. Proceedings of the $34^{\text {th }}$ EPS Conference on Plasma Physics, P2.039, Warsaw, 2007.

[14] J. D. Callen et al. Nucl. Fusion, 50:064004, 2010.

[15] P. J. Catto et al. Plasma Phys. Control. Fusion, 55:045009, 2013.

[16] C. S. Chang. Phys. of Plasmas, 4:2241, 1997. 
[17] E. Viezzer et al. Rev. Sci. Instrum., 83:103501, 2012.

[18] A. Mlynek et al. Rev. Sci. Instrum., 81:033507, 2010.

[19] M. Willensdorfer et al. Plasma Phys. Control. Fusion, 56:025008, 2014.

[20] B. Kurzan et al. Rev. Sci. Instrum., 82:103501, 2011.

[21] W. Suttrop. Practical Limitations to Plasma Edge Electron Temperature Measurements by Radiometry of Electron Cyclotron Emission. IPP Report 1/306, Max-Planck-Institute for Plasma Physics, Garching, Germany, 1997.

[22] R. Fischer et al. Fus. Sci. Technol., 58:675, 2010.

[23] A. Burckhart et al. Nucl. Fusion (accepted for publication).

[24] J. X. Rossel et al. Nucl. Fusion, 52:032004, 2012.

[25] E. Poli et al. Comp. Phys. Commun., 136:90, 2001.

[26] A. Burckhart et al. Plasma Phys. Control. Fusion, 52:105010, 2010.

[27] T. Pütterich et al. Phys. Rev. Lett., 102(025001), 2009.

[28] G. V. Pereverzev et al. IPP Report (5/98), 2002.

[29] C. Angioni et al. Phys. Plasmas, 7:1224, 2000.

[30] A. G. Peeters. Phys. Plasmas, 7(1):268, 2000.

[31] E. A. Belli and J. Candy. Plasma Phys. Control. Fusion, 50:095010, 2008.

[32] W. A. Houlberg et al. Phys. Plasmas, 4(9):3230, 1997. 